\title{
Goal Programming - The Means for Optimization of Sales Allocation
}

\author{
V. Kesava Reddy ${ }^{1}$, Dr. S. V. Sunitha ${ }^{2}$, Dr.Y.Raghunath Reddy ${ }^{3}$ \\ ${ }^{1}$ Research Scholar, Department of Mathematics Rayalaseema University, Kurnool, A.P. India. \\ ${ }^{2}$ Asst. Professor, Department of Mathematics Rayalaseema University, Kurnool, A.P. India. \\ ${ }^{3}$ Asst. Professor, Department of OR \& SQC Rayalaseema University, Kurnool, A.P.India.
}

\begin{abstract}
Goal programming is a branch of multi-objective optimization, which in turn is a branch of multicriteria decision analysis, which is an optimization programming. Goal programming models are very similar to linear programming models whereas linear programs have one objective and goal programming can have several objectives. One of the most difficult decision problems in marketing is the determination of optimum sales allocation as a part among sales of report. This paper presents the development process of a goal programming model for the resolution of sales policy which includes all exclusive product line sold in numerous sales territory.
\end{abstract}

Keywords: Goal programming model, Sales allocation

\section{Introduction}

After the II world war, the Industrial world faced a depression and to solve the various industrial problems. Industrialist tried the models, which were successful in solving their problems. Industrialist learnt that the techniques of Operation Research can conveniently apply to solve industrial problems. Then onwards, various models of Operation Research/Goal Programming have been developed to solve industrial problems. In fact GP models are helpful to the managers to solve various problems; they face in their day to day work. These models are used to minimize the cost of production, increase the productivity and use the available resources carefully and for healthy industrial growth. One of the most difficult decision problems in marketing is the determination of the optimum allocation of sales effort among the various market elements. As numerous factors used the complex relationship among these factors, it is often beyond the ability of the sales manager to identify the ideal allocation alternative. Thus it has become crucial for the retail manager to estimate the capabilities and applications of the various quantitative of management science techniques so that he can thoroughly evaluate its alternative allocation opportunities. Generally, wages represent more than half of the total expenses in retailing. Being single and effective, sales scheduling is one of the most important jobs for the retail manager.

The development has pointed out the requirement of some efficient manpower scheduling as a means to the optimum sales effort allocation. The objective of this paper is directed to the determination of a sales policy which includes all exclusive product line sold in numerous sales territories.

Benayoun et.al[1] have developed the Linear programming with multiple objective functions. Buchanan [2] has developed with naïve approach solving problem. Caballero et.al [3] have developed efficient solution in convex multi objective programming. Caballero et.al [4] have divised interactive multiple objective methods to determine the budget assignment to the hospitals of a sanitary system. Caballero et.al [5] have discussed interactive system for multi objective programming. Korhonen et.al [6] have proposed interactive method for solving the multiple criteria problem. Miettinen [7] has described non linear multi objective optimization. Miettinen et.al [8] have developed Interactive bundle-based method for nondifferentiable multi-objective optimization.Miettinen et.al [9] have developed On scalarizing functions in multiobjective optimization. Miettinenk et.al [10]have organized synchronous approach in interactive multiobjective optimization. Miettinenk et.al [11] have discussed experiments with classification based on scalarizing functions and interactive multiobjective optimization. Sawaragi et.al [12] have proposed theory of multiobjective Optimization. Steuer [13] has proposed an interactive multiple objective linear programming procedure. Steuer et.al [14] have developed multiple Criteria Optimization in theory of computation and application. Wierzbieki [15] has described basic properties of scalarizing functional for multiobjective optimization. 


\section{Data of The Problem}

The Jewellery Company markets its product line through 30 salesmen, is as follows: The bulk of the firms annual sales, however, is made by only seven salesmen operating in seven separate sales territories. The firm sells nine product lines of merchandise. The required information is given in Table 1, where each column shows how a rupee in sales in each of the seven territories is distributed among the various product lines in 2012. For example $0.04 \mathrm{Co}$-efficient for the first product (belt) indicates that on the average of centres of every rupee of firms merchandise sold in territory No. 1 is spent on their items.

Table1: Rupee Value of Sales for a Product in Territories (2012)

\begin{tabular}{|l|c|c|c|c|c|c|c|}
\hline Product lines & No.1 & No.2 & No.3 & No.4 & No.5 & No.6 & No.7 \\
\hline Belts & 0.05 & 0.04 & 0.03 & 0.18 & 0.20 & 0.16 & 0.002 \\
\hline Buckles & 0.07 & 0.03 & 0.03 & 0.15 & 0.16 & 0.09 \\
\hline Package Goods & 0.23 & 0.38 & 0.32 & 0.26 & 0.27 & 0.00 \\
\hline Necklaces & 0.06 & 0.06 & 0.06 & 0.12 & 0.18 & 0.11 & 0.60 \\
\hline Ear Rings & 0.18 & 0.18 & 0.18 & 0.18 & 0.18 & 0.18 & 0.18 \\
\hline Bracelets & 0.15 & 0.25 & 0.15 & 0.15 & 0.15 & 0.15 & 0.08 \\
\hline Gold Store & 0.17 & 0.15 & 0.20 & 0.12 & 0.08 & 0.08 & 0.17 \\
\hline Hematitie & 0.20 & 0.09 & 0.20 & 0.04 & 0.04 & 0.15 & 0.13 \\
\hline Job Turquite & 0.04 & 0.3 & 0.3 & 0.3 & 0.3 & 0.3 & 0.3 \\
\hline
\end{tabular}

The market potential for each sales territory for the planning period is given in Table 2 :

Table 2 : Marketing Potential in Each of Seven Selling Areas (2012)

\begin{tabular}{|c|c|}
\hline Territory No. & Market Potential (Rs) \\
\hline 1 & $3,50,000.00$ \\
\hline 2 & $2,50,000.00$ \\
\hline 3 & $2,25,000.00$ \\
\hline 4 & $3,25,000.00$ \\
\hline 5 & $2,00,000.00$ \\
\hline 6 & $2,35,000.00$ \\
\hline 7 & $2,20,000.00$ \\
\hline Total & $\mathbf{1 8 , 0 5 , 0 0 0 . 0 0}$ \\
\hline
\end{tabular}

Table3 represents the maximum amount of product line capacity that is available to the firm at the given price structure of nine products lines. We assume that the firm can acquire sufficient working capital to handle Rs. $18,05,000$ in sales for the 2012 planning period.

Table 3 : Maximum Amount of Product Line Capacity (2012)

\begin{tabular}{|c|c|}
\hline Lines & Product Line Capacity $(\mathbf{R s})$ \\
\hline 1 & $\mathbf{9 0 , 0 0 0 . 0 0}$ \\
\hline 2 & $\mathbf{4 0 , 0 0 0 . 0 0}$ \\
\hline $\mathbf{3}$ & $\mathbf{3 , 2 0 , 0 0 0 . 0 0}$ \\
\hline $\mathbf{4}$ & $\mathbf{9 0 , 0 0 0 . 0 0}$ \\
\hline $\mathbf{5}$ & $\mathbf{2 , 7 5 , 0 0 0 . 0 0}$ \\
\hline $\mathbf{6}$ & $\mathbf{2 , 2 5 , 0 0 0 . 0 0}$ \\
\hline $\mathbf{7}$ & $\mathbf{2 , 5 0 , 0 0 0 . 0 0}$ \\
\hline $\mathbf{8}$ & $\mathbf{2 , 5 0 , 0 0 0 . 0 0}$ \\
\hline
\end{tabular}

\section{The Model}

\subsection{GOAL PROGRAMMING MODEL}

The general GP model can be defined as follows.

Minimize $\sum \mathrm{W}_{\mathrm{J}} \mathrm{P}_{\mathrm{J}}\left(d_{I}^{-}+d_{I}^{+}\right)$

Subject to $\sum\left[\mathrm{A}_{\mathrm{IJ}} \mathrm{X}_{\mathrm{IJ}}\right]+\mathrm{I} d_{I}^{-}-\mathrm{I} d_{I}^{+}=\mathrm{B}_{\mathrm{I}}$

Where $\mathrm{W}_{\mathrm{J}}$ is the preemptive weight of each priority $\mathrm{J}, \mathrm{P}_{\mathrm{J}}$ is the preemptive priority of goal $\mathrm{J}$ or constraint $\mathrm{J}, \mathrm{A}_{\mathrm{JJ}}$ is the technological coefficient between decision variable $\mathrm{I}$ and constraint $\mathrm{J}, \mathrm{X}_{\mathrm{IJ}}$ is the decision variable $\mathrm{I}$ on constraint $\mathrm{J}, d_{I}^{+}$is positive deviation variable, $d_{I}^{-}$is Negative deviation variable, and $\mathrm{B}_{\mathrm{I}}$ is the right-handside value of constraint $\mathrm{I}$. 


\subsection{Goal Constraints}

Belt Capacity :

$0.05 \mathrm{x}_{1}+0.04 \mathrm{x}_{2}+0.03 \mathrm{x}_{3}+0.18 \mathrm{x}_{4}+0.20 \mathrm{x}_{5}+0.16 \mathrm{x}_{6}+0.002 \mathrm{x}_{7}+\mathrm{d}_{1}{ }^{-} \mathrm{d}_{1}{ }^{+}=90,000$

Bulk Capacity :

$0.07 \mathrm{x}_{1}+0.03 \mathrm{x}_{2}+0.03 \mathrm{x}_{3}+0.15 \mathrm{x}_{4}+0.16 \mathrm{x}_{5}+0.09 \mathrm{x}_{6}+0.0 \mathrm{x}_{7}+\mathrm{d}_{2}{ }^{-}-\mathrm{d}_{2}{ }^{+}=40,000$

Package Goods :

$0.23 \mathrm{x}_{1}+0.38 \mathrm{x}_{2}+0.32 \mathrm{x}_{3}+0.26 \mathrm{x}_{4}+0.27 \mathrm{x}_{5}+0.27 \mathrm{x}_{6}+0.60 \mathrm{x}_{7}+\mathrm{d}_{3}{ }^{-}-\mathrm{d}_{3}{ }^{+}=3,20,000$

Necklaces :

$0.06 \mathrm{x}_{1}+0.06 \mathrm{x}_{2}+0.06 \mathrm{x}_{3}+0.12 \mathrm{x}_{4}+0.18 \mathrm{x}_{5}+0.11 \mathrm{x}_{6}+0.05 \mathrm{x}_{7}+\mathrm{d}_{4}^{-}-\mathrm{d}_{4}{ }^{+}=90,000$

Ear Rings :

$0.18 \mathrm{x}_{1}+0.18 \mathrm{x}_{2}+0.18 \mathrm{x}_{3}+0.18 \mathrm{x}_{4}+0.18 \mathrm{x}_{5}+0.18 \mathrm{x}_{6}+0.18 \mathrm{x}_{7}+\mathrm{d}_{5}{ }^{-}-\mathrm{d}_{5}{ }^{+}=2,25,000$

Bracelets :

$0.15 \mathrm{x}_{1}+0.25 \mathrm{x}_{2}+0.15 \mathrm{x}_{3}+0.15 \mathrm{x}_{4}+0.15 \mathrm{x}_{5}+0.15 \mathrm{x}_{6}+0.08 \mathrm{x}_{7}+\mathrm{d}_{6}{ }^{-}-\mathrm{d}_{6}{ }^{+}=2,50,000$

Gold Stone :

$0.17 \mathrm{x}_{1}+0.15 \mathrm{x}_{2}+0.20 \mathrm{x}_{3}+0.12 \mathrm{x}_{4}+0.08 \mathrm{x}_{5}+0.08 \mathrm{x}_{6}+0.17 \mathrm{x}_{7}+\mathrm{d}_{7}^{-}-\mathrm{d}_{7}{ }^{+}=2,50,000$

Hematite :

$0.20 \mathrm{x}_{1}+0.09 \mathrm{x}_{2}+0.20 \mathrm{x}_{3}+0.04 \mathrm{x}_{4}+0.04 \mathrm{x}_{5}+0.15 \mathrm{x}_{6}+0.13 \mathrm{x}_{7}+\mathrm{d}_{8}{ }^{-}-\mathrm{d}_{8}{ }^{+}=2,50,000$

Job turquite :

$0.04 \mathrm{x}_{1}+0.3 \mathrm{x}_{2}+0.3 \mathrm{x}_{3}+0.3 \mathrm{x}_{4}+0.3 \mathrm{x}_{5}+0.3 \mathrm{x}_{6}+0.3 \mathrm{x}_{7}+\mathrm{d}_{9}{ }^{-} \mathrm{d}_{9}{ }^{+}=60,000$

Market Potential Goals for Territory 1-7 are as follows :

$\begin{array}{rlll}\mathrm{X}_{1}+\mathrm{d}_{10}{ }^{-}-\mathrm{d}_{10}{ }^{+} & = & 3,50,000 \\ \mathrm{X}_{2}+\mathrm{d}_{11^{-}}-\mathrm{d}_{11}{ }^{+} & & = & 2,50,000 \\ \mathrm{X}_{3}+\mathrm{d}_{12}{ }^{-}-\mathrm{d}_{12}{ }^{+} & = & 2,25,000 \\ \mathrm{X}_{4}+\mathrm{d}_{13^{-}}-\mathrm{d}_{13}{ }^{+} & = & 3,25,000 \\ \mathrm{X}_{5}+\mathrm{d}_{14^{-}}-\mathrm{d}_{14}{ }^{+} & = & 2,00,000 \\ \mathrm{X}_{6}+\mathrm{d}_{15}{ }^{-}-\mathrm{d}_{15}{ }^{+} & = & 2,35,000 \\ \mathrm{X}_{7}+\mathrm{d}_{16^{-}}-\mathrm{d}_{16}{ }^{+} & & = & 2,20,000\end{array}$

\subsection{Objective function:}

Mathematically the G.P. model can be developed as follows:

Minimize $\mathrm{Z}=\sum \mathrm{p}_{\mathrm{i}} \mathrm{d}_{\mathrm{i}}^{+}+\sum \mathrm{p}_{\mathrm{i}} \mathrm{d}_{\mathrm{i}}{ }^{-}$

$$
\mathrm{i}=1 \quad \mathrm{i}=10
$$

\section{Result And Discussion}

The solution obtained by using the QSB+ computer software may be interpreted as follows:

Table 4 : Result Analysis

$$
\begin{aligned}
& \mathrm{x}_{1}=\mathrm{x}_{2}=\mathrm{x}_{3}=0 ; \mathrm{x}_{4}=0 ; \mathrm{x}_{5}=2,25,000 \\
& \mathrm{x}_{6}=1,00,000 ; \mathrm{x}_{7}=2,13,000 \\
& \mathrm{~d}_{1}^{+}=39,000 ; \mathrm{d}_{1}^{-}=0 ; \mathrm{d}_{2}^{+}=\mathrm{d}_{2}^{-}=0 \\
& \mathrm{~d}_{3}^{+}=\mathrm{d}_{3}^{-}=0 ; \mathrm{d}_{4}^{+}=16,000 ; \mathrm{d}_{4}^{-}=0 \\
& \mathrm{~d}_{5}^{+}=61,000 ; \mathrm{d}_{5}^{-}=0 ; \mathrm{d}_{6}^{+}=16,000 ; \mathrm{d}_{6}^{-}=0 \\
& \mathrm{~d}_{7}^{+}=60,000 ; \mathrm{d}_{7}^{-}=0 ; \mathrm{d}_{8}^{+}=65,000 ; \mathrm{d}_{8}^{-}=0 \\
& \mathrm{~d}_{9}^{+}=\mathrm{d}_{9}^{-}=\mathrm{d}_{10}^{+}=\mathrm{d}_{10}{ }^{-} ; \mathrm{d}_{11}^{+}=1,00,000 ; \mathrm{d}_{11}{ }^{-}=0 \\
& \mathrm{~d}_{12}{ }^{+}=70,000, \mathrm{~d}_{12}{ }^{-}=0 ; \mathrm{d}_{13}{ }^{+}=0 ; \mathrm{d}_{14}{ }^{+}=600,000 \\
& \mathrm{~d}_{14}{ }^{-}=0 ; \mathrm{d}_{15}{ }^{-}=\mathrm{d}_{15}=\mathrm{d}_{16}{ }^{-}=\mathrm{d}_{16}{ }^{+}=0
\end{aligned}
$$

\section{Conclusion}

We notice that the optimal solution excludes the sales territories No.5 and 4 and unless management has some reasons for including these sales areas. It would be more beneficial to drop them. Selling in territory 1 and 4 will only result in a decline in net revenue. 


\section{References}

[1]. Benayoun, R., Montgolfier J., Tergny, J., Laritchev,O., 1971.Linear programmingwith multiple objective functions :Step method (STEM) Mathematical programming 1,366-375.

[2]. Buchanan, J.T., n1997. A naïve approach for solving MCDM problems:The GUESS method. Journal of the Operational Research Society 48,202-206.

[3]. Caballero, R., Rey, L., Ruiz, F., 1996.Determination of satisfying and efficientsolutions in convex multi-objective programming. Optimization 37, 125-137.

[4]. Caballero, R., Gomez, T., Lopez del Amo, M.P., Luque, M., Martin, J., Ruiz, F., 2002a. Using interactive multiple objective methods to determine the budget assignmentto the hospitals of a sanitary system. In: Trzaskalik, T. (Ed), Multiobjective and Goal programming. Springer-Verlag, Berling, pp. 209-220.

[5]. Caballero, R., Luque, M., Molina, J., Ruiz, F., 2002b. PROMOIN:An interactivesystem for multiobjective programming.International Journal On InformationTechnologies and Decision Making 1, 635-656.

[6]. Korhonen, P., Laakso, J., 1986. A visual interactive method for solving the multiplecriteria problem (VIA).Europeam Journal of Operational Research 24, 277-287.

[7]. Miettinen, K., 1999.Nonlinear Multiobjective Optimization.Kluwer AcademicPublishers, Massachusetts.

[8]. Miettinen, K., Makela, M.M., 1995.Interactive bundle-based method fornondifferentiable multi-objective optimization:NIMBUS Optimization 34, 231-246.

[9]. Miettinen, K., Makela, M.M., 2022. On scalarizing functions in multiobjectiveoptimization. Or Spektrum 24, $193-213$.

[10]. Miettinenk, K., Makela, M.M., 2006.Synchronous approach in interactivemultiobjective optimization. European Journal of Operational Research 170, 909-922.

[11]. Miettinenk, K., Makela, M.M., Kaario, K., 2006.Experiments with classificationbased scalarizing functions interactive multiobjective optimization .European Journal ofOperational Research 175, 931-947.

[12]. Sawaragi, Y., Nakayama, H., Tanino, T., 1985.Theory of Multiobjective Optimization.Academic press, New York.

[13]. Steuer, R.E., 1977.An interactive multiple objective linear programming procedure.TIMS Studies in Management Science 6, 225239 .

[14]. Steuer, R.E., 1986.Multiple Criteria Optimization: Theory, Computation andApplication. Wiley Series in Probability and Mathematical Statistic-Applied, New York.

[15]. Wierzbieki, A.P., 1977.Basic properties of scalarizing functional for multiobjectiveoptimization. Optimization 8, 55-60. 